\title{
New developments in photochemical technology
}

\author{
Jean-Claude André ${ }^{(1)}$, Marie-Laure Viriot ${ }^{(1)}$ and Jacques Villermaux (2) \\ (1) GRAPP, U.A. 328-CNRS, (2) LSGC-CNRS, ENSIC-INPL, 1, rue Grandville, \\ 54042 Nancy Cedex, France.
}

\begin{abstract}
Photochemistry and photophysics can be regarded as the natural phenomena occurring in the presence of light in the "space-time frequency" space with the predominant function - to obtain chemical products and/or objects. In this article two critical points are developed: (1) how to set up a new photochemical reaction at the laboratory scale (feasibility stage) and then (ii) how to increase the yield of reaction, trying to reach the ind ustrial synthesis scale. To start with it needs to be determined: what is the optimal source? which is the best reactor? and which are the wavelengths to be filtered and when (photochemical engineering)? The phototransformation of hop $\alpha$-acids into iso- $\alpha$-acids, for possible application in brewery, is described as an example.
\end{abstract}

\section{INTRODUCTION}

In 1933, Emschwiller wrote the following sentence relative to photochemistry (ref. 1): "The possibilities of photochemistry are numberless. Photochemical reactions are multiple and varied, as well as chemical reactions are. Light allows new transformations, increases the rate of already known reactions, improves their selectivity... In organic chemistry, in the case of many photolysis, light is operative as an universal destroying agent... Light is also a synthetic agent. It can create and destroy the same compounds... New compounds can be create by various reactions, such as halogenations, oxidations, reductions, hydrolysis, polymerisations and so on".

Besides this positive point of view, relative to the comprehension of the transformation of the matter, Emschwiller had a pessimistic view of the future of photochemistry if applied in the industry : high cost and large installations would be necessary to prepare compounds on a large scale and consequently there is no surprise if there are only few examples of practical applications of photochemistry (ref. 2,3 ).

In fact, photochemistry and photophysics are two complementary young sciences. They have only got their own fields for the last decades for theoretical and technical reasons. These two sciences correspond to the description of natural phenomena in the presence of light, in the "space-time frequency" space. The purposes of these descriptions are to point out the laws hidden behind these phenomena.

Actually, the purposes of these sciences are to obtain chemical products or objects, and their developments are based on a different conceptual analysis such as technological knowledge.

In order to realize this, the modelisation of photochemical phenomena is used. The modelisation which is based on one hand on physical and chemical knowledge and mathematics on the other hand is not only an important approach for a technological development, but also needs two parallel ways of research which are : (i) the conception coming from the imagination corrected by the knowledge of the reality and of what is possible and (ii) the experiments which allow the comparison between the theory and the experimentation.

A11 these considerations must allow answer to the two following questions : (i) how to set up a new photochemical reaction in a laboratory (feasibility stage) and (ii) how to increase the amount of product made, trying to reach the industrial scale.

We try to show hereafter how to do it : what are the concepts and the methods to be used to reach these two aims. However if for some reactions it is possible to set up complex reactor/source systems, we have decided only to describe the basic concepts, which may seem elementary to the specialist but which from our view point need to be defined. That way, we hope that if someone wants to set up a new synthesis, he will know the limits but also the possibilities of this technology as well as he knows the other techniques. 


\section{HOW TO SET UP A NEW PHOTOCHEMICAL REACTION IN A LABORATORY}

In contrast to classical chemistry (ground states of the molecules) photochemical reactions (excited states of the molecules) involve light as the principal reactive, which generally comes from outside the reaction medium (with an exception : chemiluminescence).

By "synthetic photochemistry", we mean any type of process which involves light to transform products into other products. Consequently, a photochemical reaction will generally associate the following units : reactives and solvents, light sources, reactor, reaction course (ref. 4).

To set up a new photochemical reaction, the knowledge of photochemical processes already described in the literature is supposed to be known (books or annual reviews). In any case, to develop a process in a synthesis plant, photochemistry will be prefered to classical chemistry only if all the parameters playing an essential rôle have already been carefully studied in the laboratory.

\subsection{Feasibility stage: the choice of reactive(s) and solvent(s)}

Let us consider the transformation of a compound $A_{1}$, in the possible presence of other compounds $A_{i}(i \neq 1)$ by irradiation with ultra-violet or visible light, in a batch reactor, at the beginning of the reaction. The previous knowledge of physico-chemical characteristics allow to determine under which conditions the reaction $A_{i} \rightarrow A_{j}$ can occur (or be selected).

Let us remember that there are two ways to realize the excitation of a molecule : (i) a direct excitation, by absorption of a photon, which wavelength lies inside the limits of the absorption spectrum of the compound $\mathrm{A}_{1}$ and (ii) an indirect way (sensitization or catalysis), by an energy or an electron transfer from a molecule donor $D$ to the acceptor $A_{1}$.

The investigation of the photochemical reaction of the compound $A_{1}$ will need the four following studies :

1.1.1. Absorption spectrum of the compound $\mathrm{A}$

The absorption spectra of the compounds must be known prior to the setting up of a photoreaction : (i) by direct irradiation : to choose the light source and (ii) by indirect irradiation : to choose the kind of the donor required and its concentration, that the donor will absorb the major part of the emitted light.

Moreover the absorption spectra give information on the nature of transitions involved by the light absorption and also on the possible associations in the ground state. Solvents and temperature effects can also give information on the nature and efficiency of electronic transitions.

1.1.2. Determination of the photophysical processes (fluorescence, phosphorescence) and the primary photochemical processes

The three following points can be studied :

(i) nature of the emission : the emission can be a fluorescence (emission between singlet states) and/or a phosphorescence (emission from a triplet to a singlet state). Lifetimes and quantum efficiencies $\left(\phi_{f}\right.$ and $\left.\phi_{p h}\right)$ can be determined. Emission spectra are related to the electronic configurations of the excited emitting states. It is important to know the values of $\phi_{f}$ and $\phi_{p h}$ because they correspond to the emitted light energies lost for the photoreac-

(ii) nature of the chemical intermediates and their lifetimes : chemical intermediates directly generated by primary photochemical processes are related to the possible mechanism of the transformation. Different methods or techniques can be used to detect these intermediates. For example : trapping the species in chemical reactions (indirect way) and/or direct detection by use of physical techniques : flash photolysis, low temperature studies in a rigid matrix with simultaneous detection (IR, UV, RMN), ESR (electron spin resonance) for the detection of monoradicals and triplet states, CIDNP (chemically induced dynamic nuclear polarisation) for the detection of diradicals and pair of radicals.

(iii) kinetics of the reaction : this corresponds to the determination of quantum yield of photophysical and photochemical processes, rates of reactions, lifetimes of excited states (singlet state : single photon counting experiments, triplet state : measurements based on energy transfers, flash photolysis...).

Please remember that the quantum yield of formation of a product $A_{j}\left(\phi_{j}\right)$ is expressed by the following equation :

$$
\phi_{j}=\frac{\text { number of molecules of } A_{j} \text { formed }}{\text { number of photons absorbed by } A_{1}}
$$


The determination of $\phi_{j}$ needs two measurements : first the light intensity corresponding to the number of absorbed photons in the reaction volume by unit of time : $I$, by an actinometric method (ref. 5), secondly : the number of $A_{j}$ molecules formed, which needs a quantitative analytical method (IR, UV, RMN, CpV, hplc, ...). This method must be very accurate especially if the yields are low, because it is often necessary to stop the reaction at low conversion yields, to prevent internal filter and secondary reactions. The knowledge of $\phi_{j}$ is essential for the determination of the time of reaction and as a consequence for the cost of the reaction even if the chemical yield of reaction is as high as possible.

The study of the influence of the absorbed light intensity $\left(I_{2}\right)$ on the kinetics of the phototransformation can give information on the reaction mechanism : mono or biphotonic processes, radical chain reactions (usually $I_{a}$ )...

\subsubsection{Structural rearrangement due to the transformation}

To elucidate the reaction mechanism, it can be necessary to perform the following experi-

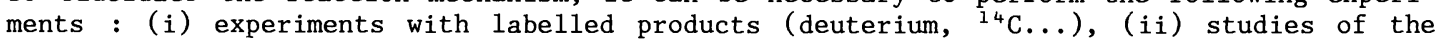
stereochemistry of the products (related to the symmetry rules), (iii) influence of different substituents on the product $A_{1}$ and (iv) influence of steric factors.

In fact, a reaction mechanism only represents a possibility within several hypothesis based on validation, but never is a certainty (ref. 4).

\subsubsection{Influence of the macroscopic parameters}

The following influences can be taken into account : temperature (activation energy), solvent (viscosity, dielectric constant, polarity, hydrogen donor, as a reactive, solvatation, ionic strength...), $\mathrm{pH}$ (ionic forms in ground and excited states), pressure (viscosity, activation volume), phase (solid, liquid, gaseous, adsorbed on a solid, matrix, supercritic fluid, interfaces...), reactant concentration (important for the dimerisations), oxygen (as a quencher, as an oxidative agent), light intensity and its profile, stirring of solutions (this can allow the solution homogeneous in instable species having short lifetimes such as free radicals), turbidity and so on.

\subsection{Problems in having a photoreactor adapted to the reaction}

In practice, the studies described in section 1.1. can be performed with classical light sources and batch/flow reactors (ref. 6). It can be necessary to use sufficiently low concentrations of the products to get a quasi-homogeneous absorption of the light by the absorbing substance. Moreover as well as in classical chemistry a differential reactor will be used to get uniform and well-controlled concentrations and temperature.

This situation reflecting some simple cases : $A_{1} \rightarrow A_{j}$ in which $A_{j}$ doesn't absorb the considered wavelength (photoreduction of ketones, for example) will ${ }^{\mathrm{j}}$ represent the ideal case, easily extendable to industrial or synthesis plants. On the contrary, it might be more difficult to consider a "good reactor" for some more complex reactions as the following ones : ( $i$ ) the $\mathrm{A}_{\mathrm{j}}$ formed absorb the emitted light, (ii) the photochemical reaction is a dimerisation which implies a high concentration of $\mathrm{A}_{1}$, leading to a possible non-homogeneous absorption, (iii) the order of the reaction related to absorbed light intensity is different from one, and so on...

For example, one can consider radical chain photochlorations, for which kinetics are so fast that they are limited by transport processes of chlorine (ref. 7). One can also consider another reaction, studied by our group, for its potential application in brewery : the phototransformation of hop products, i.e. the photoisomerisation of humulone $\left(\alpha-a c i d, A_{1}\right)$ into trans-iso-humulone (iso- $\alpha$-acid, $\mathrm{A}_{2}$ ).

Here we will use this reaction as an typical example to show the influence of reactive stirring (ref. 10):

$$
\mathrm{A}_{1} \stackrel{\mathrm{h} v}{\rightarrow} \mathrm{A}_{2} \stackrel{\mathrm{h} v}{\longrightarrow} \mathrm{A}_{3}
$$

Here also the purpose of stirring is not to get homogeneous concentrations of the transient species (electronic excited states, free radicals, ...) but of the molecular reactives.

If the absorption of the light by $A_{1}$ is not homogeneous, then Fig. 1 represents the variations of local concentrations of $\mathrm{A}_{2}$ as a function of time and distance between this point and the entrance of the light into the reactor. Close to the entrance, one observes that the formation of $\mathrm{A}_{2}$ which leads to photoproducts, caused by the lack of stirring. Subsequently, wrong conclusions relative to the kinetics and mechanims could be made in such a case if this phenomenon is not taken into account. 


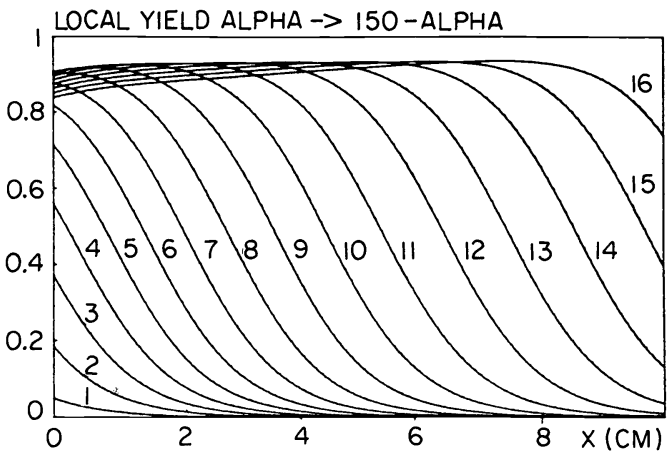

Fig. 1. Visualization of the effect on nonmixing of irradiated solutions on the yield of iso- $\alpha$-acids, variations of their local concentration as a function of time ( 1 to 16 a.u.) and distance.

\section{HOW TO DEVELOP PHOTOCHEMICAL SYNTHESIS}

The study presented in section 1 represents a reaction at its beginning, at low conversion yields. On the contrary, reactions for synthesis require high conversion yield with a good selectivity. Moreover, it will be useful to take economical factors into account such as the reactor cost, the product prices and so on (ref. 11). First the absorption spectra of photoproducts formed have to be studied. If there is no overlap between them and the absorption spectrum of the initial reactants, no internal filtering effect will happen. On the contrary, photoproducts can absorb the light, be photodegradable or react as sensitizer. In all these cases, they could lower the absorption by the reactants. So if $\mathrm{A}_{j}$ is a photoreactive product, one must consider the experimental conditions described in section 1 and then study the effect of $\mathrm{A}_{j}$ on the photochemical reaction.

In fact for each photochemical process one wants to develop, one carefully has to choose the source(s) and the reactor and then to decide the more appropriate reaction conditions.

\subsection{The sources}

There are now three well known types of sources with different spectral characteristics : (i) the sun which is the oldest source, with some properties of stigmatism and which has a continuous spectrum, (ii) the lamps with continuous or lines spectra, stigmatic or not, and (iii) the lasers which are often monochromatic and stigmatic.

These sources, so called primary sources can be associated with optical filters which limit their spectra. Moreover it is possible to take into account another property of some light sources which is their temporal resolution, viz. allows "non-stationnary" experiments which is a particularly promising way (i.e. multi-photonic processes).

\subsection{Reactors and photochemical engineering}

In principle, when the reactor is not already imposed, its nature, shape and use have to be computed, according to its required size. This size (from several inches to several yards) implies not only the choice of the lamp, but also all the involved transport processes.

These reactors can be batch, plug flow or perfectly stirred types, with any combination of these "ideal" reactors such as gas-liquid reactors (Fig. 2).

Their shapes in connection with the light absorption profile can allow different selectivities and an optimal use of the excitation light. This is in fact photochemical engineering, which can be defined as the method used to optimize a photochemical reaction, first the design of the reactor (type, size...) and then the optimal reaction course itself.

As well as in the other chemical reactions engineering fields, the problems are different according to the type of the reaction (continuous or not, i.e. batch or open reactor) and to the type of the medium (homogeneous, i.e. liquid or gas) generally less absorbant, or heterogeneous. This latter situation often happens because of the importance of gas-liquid chemical reactions for which one reactant is a fine dispersion of bubbles inside a liquid and sometimes is in a liquid-liquid suspension.

Let us try to analyze the specific problems involved in the development of a photochemical process. The classical steps are : (i) a priori design and size of the reactor, (ii) extrapolation, scale expansion, discontinuous/continuous modifications and (iii) running optimization of an already existing reactor.

Everybody knows that the description of the reactive system and the reactor by a mathematical model is necessary to solve the problems. In photochemical engineering, the reactor will need a uniform distribution of the light and this condition is not easy to realize. It is then necessary to know the transport properties of the reactants in the 


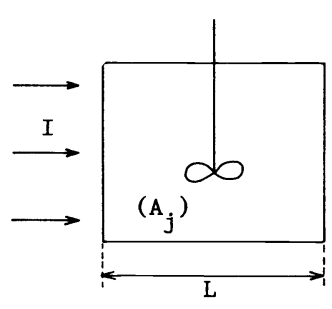

(1)

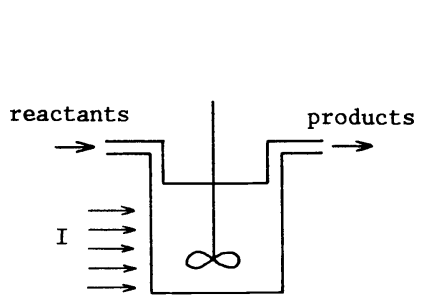

(4)

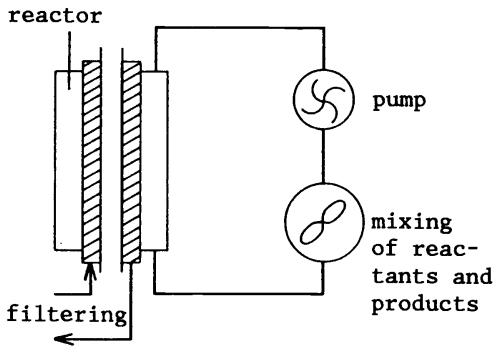

(2)

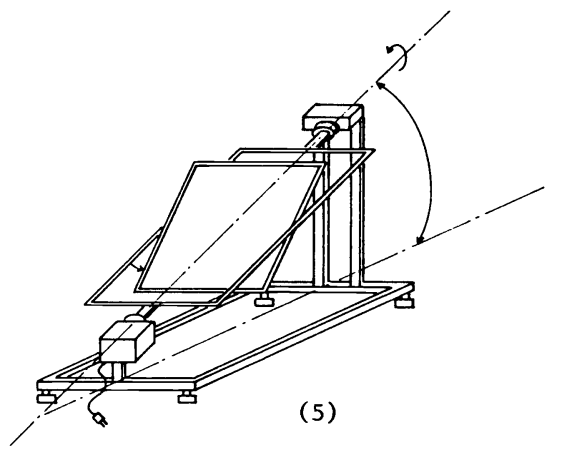

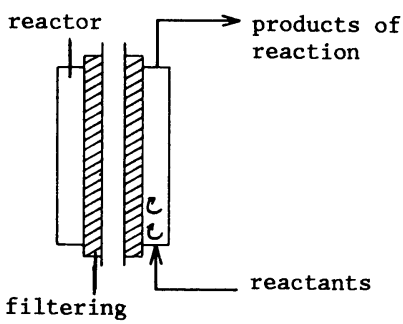

(3)

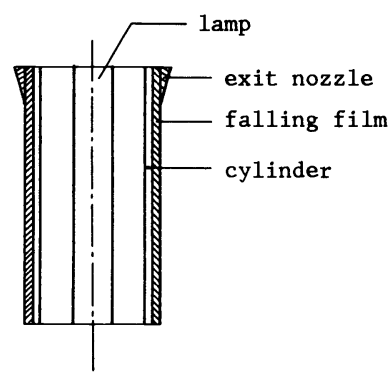

(6)

Fig. 2. Photoreactors : (1) ideal batch reactor; (2) batch, closed static system with filtering; (3) plug flow, continuous open system with filtering ; (4) ideal perfectly stirred reactor ; (5) solar plane reactor; (6) falling film reactor.

reactive medium : diffusivities, viscosities, thermal conductivities, transfer conductance at the wall. The role of the diffusivity of excited species has already been mentionned. If the correlations found in literature are not sufficient, special experiments will have to be set up to study them.

It is also particularly important to have a good model describing the light absorption inside the reactor, which of course represents an additional characteristic of photochemistry when compared to chemistry.

Finally, when these data are added to the reactive medium on a macroscopic scale (macromixing) due to the natural or forced stirring and to the fluids flows, a global mathematical model of the reactor will be obtained.

If one is interested in a local model, which will allow to compute the evolution of the reactive medium in every point, to the three balances of "classical" chemical reactors (matter, thermomechanical energy and momentum) a fourth one i.e. photonic energy has to be added (ref. 6, 12). The four equations can be written as follows :

(1) Conservation of mass of the $j$ th species : $\begin{aligned} & \text { rate of accumulation } \\ & \text { of the } j \text { th species }\end{aligned}=\begin{aligned} & \text { rate of mass of } j \\ & \text { into the reactor }\end{aligned}-\begin{aligned} & \text { rate of mass of } j \\ & \text { out of reactor }\end{aligned}+\begin{aligned} & \text { rate of generation of } \\ & j \text { due to reaction }\end{aligned}$

(2) Conservation of momentum

\begin{tabular}{|c|c|c|}
\hline of momentum & $\begin{array}{l}\text { momentum at reac- } \\
\text { tor inlet }\end{array}$ & $\begin{array}{l}\text { momentum at reac- } \\
\text { tor outlet }\end{array}$ \\
\hline
\end{tabular}

(3) Conservation of energy

$\begin{aligned} & \text { rate of accumulation } \\ & \text { of energy }\end{aligned}=\begin{aligned} & \text { rate of energy at } \\ & \text { reactor inlet }\end{aligned} \quad \begin{aligned} & \text { rate of energy at } \\ & \text { reactor outlet }\end{aligned}+\begin{aligned} & \text { rate of generation of } \\ & \text { energy due to reaction }\end{aligned}$

(4) Conservation of radiant energy at a given wavelength

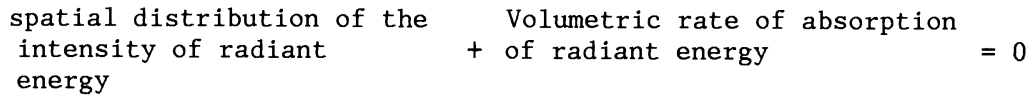


However difficulties can arise from the strong coupling existing between the mass and the radiant energy balances : the light intensity profile depends on the concentrations of the reactants and products (through absorption coefficients) and the concentrations in their turn depend on the rate reactions, i.e. on the light intensity. It is the typical description of a retroactive feedback (ref. 13). In classical reactors, such a coupling already exists between mass and heat balances, due to the rate reactions sensitivity to temperature, i.e. the thermal effects of these reactions. In a photochemical reactor a new coupling has to be added : so it has to be expected that these crossed couplings will induce instability phenomena (especially in the case of exothermic chain reactions) and difficulties to control the reactor; a study which has still to be done.

\subsection{The logical way of action in photochemical synthesis}

If the problems defined in section 1 are supposed to be solved, how does a chemist work if he wants to produce several grams of a product via a photochemical process?

Several situations may occur depending on his aims, how much time he wants to spend on this problem and what are the means he has and the one that are needed. In fact the only true question is what is the best : a reactor that is either extremely well adapted to one reaction or that is very easy to modify (flexibility). To our mind, there is no obvious answer to this important question. Moreover the type of reaction involved which can either be very simple :

$$
\left(\mathrm{A}_{1} \stackrel{\mathrm{hv}}{\rightarrow} \mathrm{A}_{2}\right)
$$

or on the contrary very complex $\left(A_{i} \underset{h \nu}{\stackrel{h \nu}{\longrightarrow}} A_{j}\right)$;

(non-monomolecular reactions, and so on) must have an important rôle in the design of the source-reactor apparatus. Sometimes, it can be necessary (excluding some sort of scientific "fashionable" behavior) to use expensive light sources such as lasers, which lead to sources-reactors systems much more difficult to set up.

In these circumstances, it does not seem possible to us to suggest a general type of action to resolve most of the problems arising in synthetic photochemistry.

Nethertheless, in some cases interesting from the view point of synthesis, we showed that it is possible to define criteria to choose the photoreactors which lead to technological innovations.

As an example, we have choosen relatively complex monomolecular reactions such as :

$$
A_{i} \stackrel{h v}{\longrightarrow} A_{j}
$$

It would have of course been possible to choose and to study other reactions as an example, such as chain reactions (ref. 14), bimolecular reactions, reactions with cage-effects, and so on...

Here we have limited ourselves to the experimental study of the humulone phototransformation under different experimental conditions, using commercial lamps of a reasonable price (mercury vapor lamps).

\section{PHOTOCHEMICAL TECHNOLOGY OF MONOMOLECULAR REACTIONS}

\subsection{Research of the most suitable wavelengths for a phototransformation}

Let us consider the a priori existence of monomolecular or pseudomonomolecular reactions of the type :

$$
A_{i} \stackrel{h v}{\longrightarrow} A_{j} \text {, }
$$

which occur in the absorption range with a quantum yield $\phi_{i j}$, independent of the wavelength. That way with the assumption that the $n A_{i}$ compounds ( $w i t h j_{i}^{\prime}=1,2, \ldots n$ ) are independent in their ensemble, the reaction system can then be defined by a (nxn) matrix $\psi=\left(\phi_{i j}\right)$. If the experimental conditions are such that the light is homogeneously absorbed in the reactor to avoid possible screen effects, it is necessary for the absorption to be low. Under these conditions, the absorptions by the different $A_{\text {a }}$ are practically independent. A computer program has been written to calculate the possible changes in the chemical reaction yields $\rho_{m}$ with the wavelength $\lambda$. This program is based on the knowledge of the variations of the molecular extinction coefficients $\varepsilon_{i}\left(A_{j}\right)$ with $\lambda$.

As an example, in Fig. 3 are shown the theoretical variations of $\rho_{m}$ with $\lambda$ in the case of the photoisomerization of humulone $\left(A_{1}\right)$ into trans-iso-humulone $\left(A_{2}\right)$ and then into unidentified products $\left(\mathrm{A}_{3}, \mathrm{~A}_{4}, \ldots\right)$ :

$$
\mathrm{A}_{1} \stackrel{\mathrm{h} \nu}{\longrightarrow} \mathrm{A}_{2} \stackrel{\mathrm{h} \nu}{\rightarrow} \mathrm{A}_{3} \stackrel{\mathrm{hv}}{\rightarrow} \mathrm{A}_{4} \stackrel{\mathrm{h} \nu}{\rightarrow}>\ldots
$$




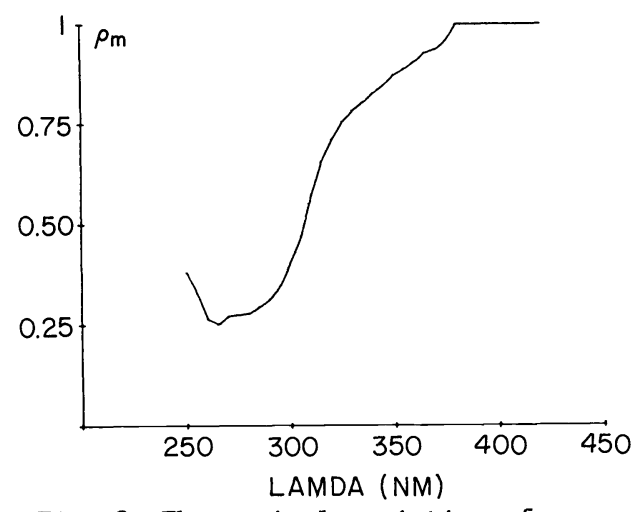

Fig. 3. Theoretical variation of $\rho$ with $(\lambda)$ for the phototransformation of humulone into trans-iso-humulone.

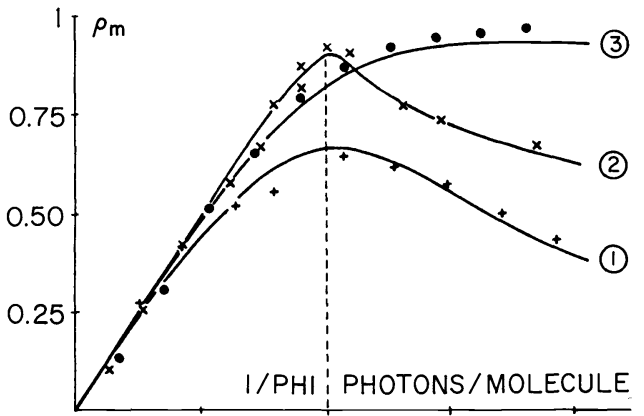

Fig. 4. Phototransformation of humulone into trans-iso-humulone, at different excitation wavelengths : (1) $313 \mathrm{~nm}$, (2) $365 \mathrm{~nm}$ and (3) $405 \mathrm{~nm}$. Comparison between the experimental data (dots) and the model (full line curves).

These variations of $\rho_{\mathrm{m}}$ vs $\lambda$ have been experimentally checked (Fig. 4) and there is a good agreement between the ${ }_{m}$ computed and experimental values.

\subsection{A new type of reactor}

The study of the influence of macroscopic factors on the system (see 1.1.4.) led us to study the influence of the viscosity of the reaction medium on the complete phototransformation of $A_{1}$ (transparency of $\left.A_{2}, A_{3} \ldots\right)$.

The humulone photoisomerization can be explained by an oxa-di- $\pi$-methane reaction (ref. 15) via the rotation of an isopropylidene group. Since this rotation occurs during the lifetime of transient species, this reaction requires the solvent cooperativity.

So the diffusional mechanism that we have developed points out the interest of a low viscosity solvent (ref. 11). This observation led us to develop a new type of photoreactor in which we used critical fluids which have a very low viscosity. This reactor (Fig. 5) was associated with a dipped lamp, which allows an efficient use of the emitted photons.

\subsection{Use of several excitation wavelengths in a plug flow reactor}

To produce compounds in small quantities (laboratory scale), it is always possible to use a quasi-monochromatic light, which generally needs a long irradiation time. That is what we did in the photoreaction of hop extracts (humulone). So the use of one radiation only 313 , 365 or $405 \mathrm{~nm}$ provided by a filtered mercury vapor lamp respectively led to an optimal yield of reaction of $0.72,0.95$ and practically 1 respectively. In these conditions, considering the high cost of the reactants, it seems better to use the longer wavelengths of the lamp (365 and $405 \mathrm{~nm}$ ) only.

However, because of the low quantum yield of the photoreaction $(\phi=0.02)$ we were interested in using the photons emitted by an industrial mercury vapor lamp in the best, most economical way. Moreover, at the beginning of the reaction, the concentration of iso- $\alpha$-acids is so low that their absorption at $313 \mathrm{~nm}$ and consequently their photodegradation is negligible. In these conditions, it is possible to realize the reaction more rapidly than with the only use of 365 and $405 \mathrm{~nm}$ lines. Then at larger conversion yield, the photodegradation of iso- $\alpha$-acids occurs by the $313 \mathrm{~nm}$ line. We shall discuss further down the stepwise transmittance filtering as shown in Fig. 6.

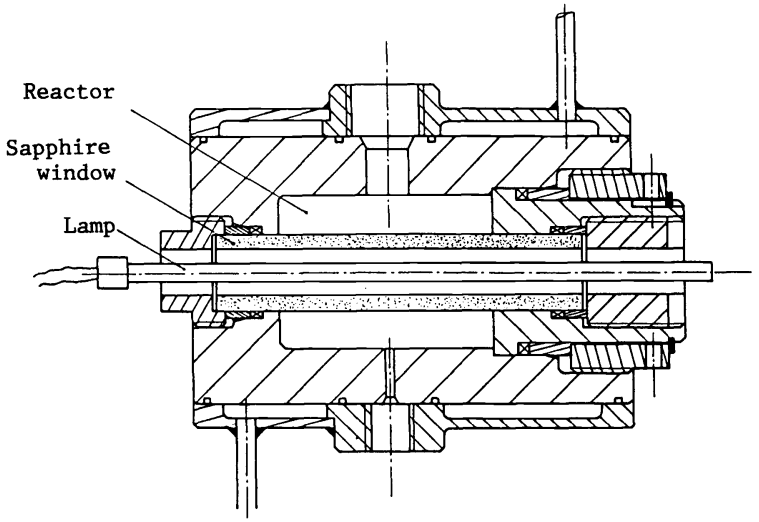

Fig. 5. Photoreactor for supercritical fluids.

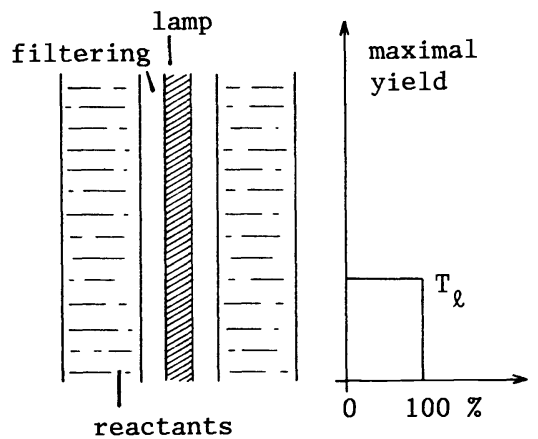

Fig. 6. Photochemical reactor with stepwise transmittance filtering at $\lambda_{\ell}$. 
3.3.1. Monochromatic source

Let us first consider either a plug flow reactor or a stirred batch reactor, associated with a monochromatic light source : the reactions have been assumed to be monomolecular or pseudomolecular :

$$
\mathrm{A}_{i} \stackrel{h v}{\longrightarrow} \mathrm{A}_{\mathrm{j}}
$$

with a quantum yield $\phi_{i j}($ with $i=1,2 \ldots, n)$ (see 3.1.).

Since one excitation wavelength is used, it is possible to express the temporal evolution of a particular compound by the relation :

$$
\frac{d\left(A_{i}\right)}{d t}=\frac{I}{D} \sum_{j=1}^{j=n} \phi_{j i} \varepsilon_{j}\left(A_{j}\right)
$$

where $I$ is the absorbed light in the reaction vessel and :

$$
D=\sum_{j=1}^{j=n} \varepsilon_{j}\left(A_{j}\right)
$$

If only one excitation wavelength is used the maximal concentration of a product $k$ from an initial mixture can be computed by the integration of $n-1$ out of the $n$ differential equations. The reaction time $\tau$ corresponds to the condition :

$$
\frac{d\left(A_{k}\right)}{d t}=0
$$

To take this equation into account a term $\mathrm{T}$ corresponding to the transmittance at the considered wavelength has to be added to the differential equations between $\left[d\left(A_{i}\right) / d t\right]$ and the macroscopic factors of the system.

So if $\frac{d\left(A_{k}\right)}{d t}>0, T=1$ and $\frac{d\left(A_{k}\right)}{d t} \leqq 0, T=0$

which leads to $\frac{d\left(A_{i}\right)}{d t}=\frac{T . I .}{D} \sum_{j=1}^{j=n} \phi_{i j} \varepsilon_{j}(A)$

3.3.2. Plurichromatic source with discrete lines

If the experimental most suitable wavelengths for the transformation can be selected, it is possible to take economical factors into account. This method is based on the fact that at the beginning of the reaction, practically all the light emitted by the source which covered the absorption spectrum of $A_{1}$ can be used. Then the rate of the reaction can be greatly improved.

If $\mathrm{m}$ wavelengths can induce the photoreaction and if $\mathrm{I}_{l}$ is the absorbed light intensity at the wavelength $\lambda_{\ell}$, then the rate of reaction of the product $i$ is :

$$
\frac{d\left(A_{i}\right)}{d t}=\sum_{\ell=1}^{\ell=m} \frac{T_{\ell} I_{\ell}}{D_{\ell}} \sum_{j=1}^{j=n} \phi_{j i} \varepsilon_{j \ell}\left(A_{j}\right) \text { with } D_{\ell}=\sum_{j=1}^{j=n} \varepsilon_{j \ell}\left(A_{j}\right)
$$

The measurements of the temporal evolution of the concentration of $A_{k}$ for an excitation at $\lambda_{\ell}$ gives the values of $\mathrm{T}_{\ell}$, so if :

$$
\left[\frac{\mathrm{d}\left(\mathrm{A}_{\mathrm{k}}\right)}{\mathrm{dt}}\right]_{\lambda_{\ell}}>0, \mathrm{~T}_{\ell}=1 \text { and if }\left[\frac{\mathrm{d}\left(\mathrm{A}_{\mathrm{k}}\right)}{\mathrm{dt}}\right]_{\lambda_{\ell}}<0, \mathrm{~T}_{\ell}=0
$$

Then it is possible to compute the optimal way of the transformation of $A_{i}$ into $A_{k}$, if $m$ wavelengths are used.

For example, in Fig. 7 are shown the transformations of $\alpha$-acids into iso- $\alpha$-acids when the following sets of wavelengths are successively used : $405-365-313 \mathrm{~nm}, 405-365 \mathrm{~nm}$ and $405 \mathrm{~nm}$ alone. The ratios of the light intensities at 405, 365 and $313 \mathrm{~nm}$ are $1: 1.64: 0.45$ 


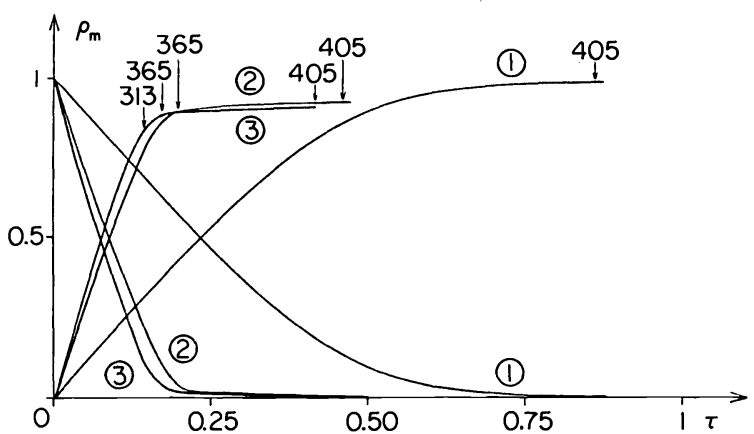

Fig. 7a. Simulation of the photo-isomerization of $\alpha$-acids into iso- $\alpha$-acids with filtering in a plug flow reactor

(1) $405 \mathrm{~nm}$; (2) 405 and $365 \mathrm{~nm}$;

(3) 405,365 and $313 \mathrm{~nm}$.

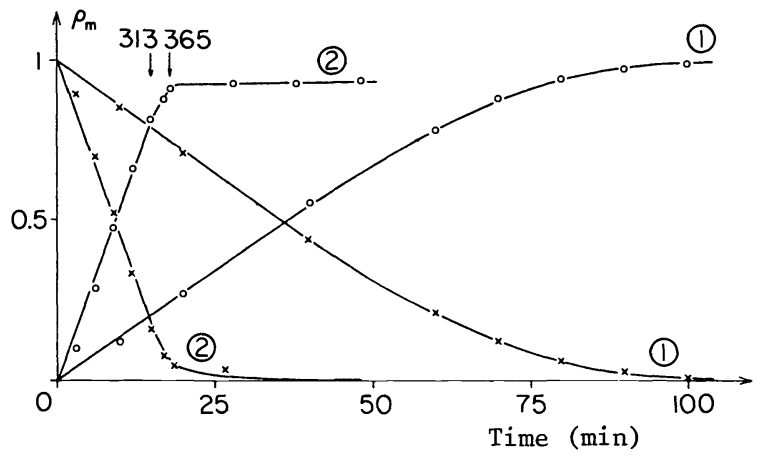

Fig. 7b. Experimental data :

(1) $405 \mathrm{~nm}$; (2) 405,365 and $313 \mathrm{~nm}$; $x, \alpha$-acids; 0 , iso- $\alpha$-acids.

respectively. In Fig. $7 \mathrm{a}$ is drawn the simulation resulting from the model previously described; the ticks correspond to the successive wavelengths filtered off. In Fig. $7 \mathrm{~b}$ are drawn the corresponding experimental results. The comparison of experiments (1) $405 \mathrm{~nm}$ only and (2) 313-365-405 nm with selective filters leads to the following conclusion : the use of the three wavelengths reduces the irradiation time by at least a factor 2 whereas the decrease of the maximal yield $(0.98$ to 0.93$)$ is only a few per cent.

\subsection{Use of a perfectly stirred reactor}

Let us first consider the use of a perfectly stirred photochemical reactor when a monochromatic light source is used, then

$$
\left(A_{i}\right)_{o}-\left(A_{i}\right)=\frac{\tau \cdot T \cdot I .}{D} \cdot \sum_{j=1}^{j=n} \phi_{i j} \varepsilon_{j}\left(A_{j}\right)
$$

where $\left(A_{i}\right)_{0}$ is the concentration of product $i$ at the reactor entrance, $\left(A_{i}\right)$ is the concentration at the reactor exit, $\tau$ is the residence time and

$$
D=\sum_{j=1}^{j=n} \varepsilon_{j}\left(A_{j}\right)
$$

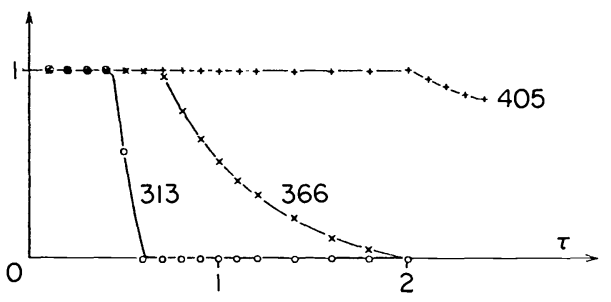

using m excitation wavelengths leads to the following expression between the reactant concentrations at the entrance and at the exit of the reactor :

$$
\begin{aligned}
\left(A_{i}\right)_{0}-\left(A_{i}\right) & =\tau \sum_{\ell=1}^{\ell=m} \frac{I_{\ell} T_{\ell}}{D_{\ell}} \sum_{j=1}^{j=n} \phi_{j i} \varepsilon_{j \ell}\left(A_{j}\right) \\
\text { with } D_{\ell} & =\sum_{j=1}^{j=n} \varepsilon_{j \ell}\left(A_{j}\right)
\end{aligned}
$$

This relationship can be solved by different mathematical methods.

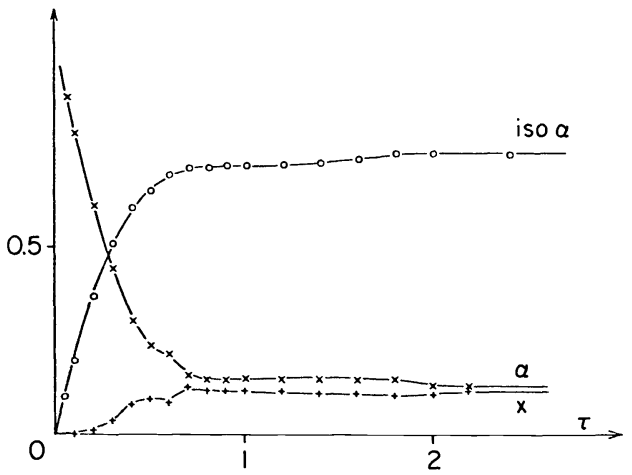

In a stirred reactor, as shown in Fig. 8, the maximum yield of the photoisomerization of $\alpha$-acids into iso- $\alpha$-acids can only be reached if only one wavelength is used. Consequently this type of reactor is unsuitable.

Fig. 8. Optimal choice of wavelengths for the photoisomerization of $\alpha$-acids into iso- $\alpha$-acids in a perfectly stirred reactor. Excitation at 405, 365 and $313 \mathrm{~nm}\left(\alpha \equiv \mathrm{A}_{1}\right.$; iso $\left.\alpha \equiv \mathrm{A}_{2} ; \mathrm{X} \equiv \mathrm{A}_{3}\right)$.

\subsection{Which type of reactor to choose?}

During this study of the photoisomerization of hop a-acids, we have thought that cutting off some wavelengths could influence the reaction, which was true. However, if this concept wants to be applied on an industrial scale, the choice of the reactor has to be carefully done. In Table 1, we have summed up the main advantages and disadvantages of some different possible systems. 
TABLE 1. Influence of the reactor on the practical application of photoisomerization of hop $\alpha$-acids.

\begin{tabular}{|c|c|c|}
\hline REACTOR & ADVANTAGES & DISADVANTAGES \\
\hline $\begin{array}{l}\text { closed : } \\
\text { static system }\end{array}$ & $\begin{array}{l}\text { stirring can take place in a } \\
\text { neighbouring tank }\end{array}$ & $\begin{array}{l}\text { - discontinous production } \\
\text { change of successives filtering solutions } \\
\text { ( } 313,365,405 \mathrm{~nm} \text { ) is necessary (it can be } \\
\text { avoided by using several consecutives batch } \\
\text { reactors) } \\
\text { use of a continuously variable filter at } \\
313 \mathrm{~nm} \text { is practically impossible. }\end{array}$ \\
\hline $\begin{array}{l}\text { open : } \\
\text { continuous flow }\end{array}$ & $\begin{array}{l}\text { continuous production } \\
\text { - use of a continuously variable } \\
\text { filter is possible } \\
\text { step transmittance is possible } \\
\text { with no filter circulation }\end{array}$ & $\begin{array}{l}\text { major difficulties occur in radial mixing of } \\
\text { the solution because of the need to displace } \\
\text { the photo-products obtained near the skin of } \\
\text { the reactor } \\
\text { in practice use of sufficiently diluted solu- } \\
\text { tion is required to avoid the skin effect. }\end{array}$ \\
\hline open : stirred & continuous production & $\begin{array}{l}\text { - low yield production } \\
\text { - only one wavelength must be used }\end{array}$ \\
\hline
\end{tabular}

So, as long as laboratory, i.e. small quantities (a few grams) of a product are required, a batch reactor can be used. The same type of reactor can also be used in an industrial plant. Thanks to the example that we presented in this paper, we have shown that it is possible to use almost any type of lamps or of reactors and that only technological and economical considerations will be the constraints to introduce in the optimization of the system.

\section{CONCLUSION}

After having defined what are the criteria to follow to have maximum of chances to produce a compound in a laboratory by a photochemical reaction, we have suggested that it is often possible to use classical reactors to develop a photochemical process, but the conditions are usually far from the optimum. If technological and economical considerations have to be taken into account (such as in the example of the photoisomerization of hop products), we have shown that it was possible to use reactors leading at the same time to a larger rate of the reaction and to a better use of the photons emitted by a light source. This kind of development could indeed be done for other examples of photoreactions.

These data clearly show that for each photoreaction it should be possible to develop an optimal reaction course and consequently an adaptated reactor. It also seems difficult to realize a simple reactor adaptable enough to be used for all the photoreactions.

Moreover photochemical engineering which was quickly mentionned here is still a broadly wide open field in which numerous fundamental problems have to be solved.

Our researches on photoreactions of industrial interest led us to contribute to this discipline, by developing new reactors and other innovative technologies, such as the use of fluorescent filtering, the use of optical fibers between the source and the reactor and the automatization of the processes.

\section{Acknowledgement}

The authors gratefully acknowledge Drs J.Y. Jezequel and A. Saĩd for helpful discussions.

\section{REFERENCES}

1. G. Emschwiller, La photochimie, Bull. Soc. Encour. Industrie Nationale, 46-53 (1933).

2. M. Fischer, Angew. Chem. Int. Ed. Eng1. 17, 16-26 (1978).

3. J.C. André, M.L. Viriot, J. Villermaux and A. Tournier, Entropie 107-108, 62-81 (1982).

4. N.J. Turro, Modern molecular photochemistry, Benjamin-Cummings, Menlo Park, CA (1978).

5. S.L. Murov, Handbook of photochemistry, Marcel Dekker, New-York (1973).

6. P.L. Yue, M. Schiavello (ed.), Photoelectrochemistry, photocatalysis and photoreactors, p. 527-547, D. Reidel (1985).

7. A. Tournier, X. Deglise and J.C. André, J. Photochem. 22, 137-155 (1983).

8. M.L. Viriot, J.C. André, M. Niclause, D. Bazard, R. Flayeux and M. Mol1, J. Inst. Brew. 86, 21-24 (1980).

9. J.C. André, M.L. Viriot, M. Niclause, D. Bazard, R. Flayeux and M. Moll, Amer. Soc. Brew. Chem. J. 38, 61-67 (1980).

10. J.C. André, M. Bouchy and J. Kossanyi, J. Photochem. 22, 213-221 (1983).

11. A. Saĩd, Thesis, Nancy (1985).

12. J. Villermaux, Génie de la réaction chimique, Lavoisier, Paris (1982).

13. A. Tournier, X. Deglise, J.C. André and M. Niclause, AIChE J. 28, 156-166 (1982).

14. J.C. André, A. Tournier and X. Deglise, J. Photochem. 22, 7-24 (1983).

15. D. de Keukeleire and G.M. Blondee1, Tetrahedron Letters, 1343-1346 (1979). 